\title{
A SUBSPACE THEOREM FOR ORDINARY LINEAR DIFFERENTIAL EQUATIONS
}

\author{
ALICE ANN MILLER
}

(Received 22 May 1989; revised 16 January 1990)

Communicated by J. H. Loxton

\begin{abstract}
The study of the $S$-unit equation for algebraic numbers rests very heavily on Schmidt's Subspace Theorem. Here we prove an effective subspace theorem for the differential function field case, which should be valuable in the proof of results concerning the $S$-unit equation for function fields. Theorem 1 states that either

has a given upper bound where

$$
\operatorname{Ord}_{a}\left(L_{1} L_{2} \cdots L_{n}\right)
$$$$
L_{1}(\mathbf{P}), L_{2}(\mathbf{P}), \ldots, L_{n}(\mathbf{P})
$$

are linearly independent linear forms in the polynomials

$$
\mathbf{P}=\left(P_{1}(x), P_{2}(x), \ldots, P_{n}(x)\right)
$$

with coefficients that are formal power series solutions about $x=0$ of non-zero differential equations and where $\operatorname{Ord}_{a}$ denotes the order of vanishing about a regular (finite) point of functions $f_{k, i}:(k=1, n ; i=1, n)$ or

$$
\mathbf{P}=\left(P_{1}(x), P_{2}(x), \ldots, P_{n}(x)\right)
$$

lies inside one of a finite number of proper subspaces of $(K(x))^{n}$. The proof of the theorem is based on the wroskian methods and graded sub-rings of Picard-Vessiot extensions developed by D. V. Chudnovsky and G. V. Chudnovsky in their function field analogues of the Roth and Schmidt theorems. A brief discussion concerning the possiblity of a subspace theorem for a product of valuations including the infinite one is also included.
\end{abstract}

1980 Mathematics subject classification (Amer. Math. Soc.) (1985 Revision): Primary $11 \mathrm{~J} 61$, $11 \mathrm{~J} 82,11 \mathrm{Q} 10$.

I would like to thank Macquarie University for their hospitality while I did the research for this paper and SERC for their financial support.

(C) 1991 Australian Mathematical Society 0263-6115/91 \$A2.00+0.00 


\section{Introduction}

For a long time there has been much interest concerning the accuracy with which algebraic numbers can be approximated by rational numbers. In 1955, K. F. Roth [6] showed that if $\alpha$ is real and algebraic of degree at least 2 then for each $\varepsilon \geq 0$ the inequality

$$
\left|\alpha-\frac{p}{q}\right|<\frac{1}{q^{2+\varepsilon}}
$$

has only many solutions in rationals $p / q$. This result is best possible of its type but non-effective in the sense that it does not yield an upper bound for the largest value of $q$. Later C. F. Osgood [4] proved an effective "Rothtype" result for the solutions of algebraic differential equations, and $D$. V. Chudnovsky and G. V. Chudnovsky [1] used wronskian methods and graded subrings of Picard-Vessiot extensions to prove an effective analogue of Roth's Theorem for the solutions of ordinary linear differential equations.

A powerful generalisation of (1) for a system of algebraic linear forms was given by Schmidt's Subspace Theorem [8] which was later extended by H. P. Schlickewei [7] to the $p$-adic case. Both results were ineffective but found to be crucial in the study of the $S$-unit equation

$$
\alpha_{1} x_{1}+\alpha_{1} x_{2}+\cdots+\alpha_{n} x_{n}=0
$$

summarized by J. H. Evertse and K. Györy [2] in their results concerning (2) for $n>2$.

The purpose of this paper is to present a proof of an effective subspace theorem for the differential function field case based on the methods used by D. V. Chudnovsky and G. V. Chudnovsky [1] in their function field analogue of Roth's Theorem.

The theorem in this paper gives a bound on a valuation of a product of linear forms $L_{1}, L_{2}, \ldots, L_{n}$ where the coefficients of each linear form are functions of $x$. As such, it is similar to a result obtained by C. F. Osgood [5, Theorem XIII]. However, the methods used by Osgood to obtain the auxillary polynomial rely on Nevanlinna theory and are therefore different from those used in this paper. Also the exceptional subspaces, though implicit in Osgood's work (the auxiliary polynomial is required to be non-zero for the bound to hold), are neither considered in detail or utilised, as they are here, to present a function field analogue of Schmidt's Subspace Theorem. 


\section{Definitions}

(1) If $y=y(x)$ is a function of $x$ then

$$
y^{(j)}=\left(\frac{\partial}{\partial x}\right)^{j}, \quad 0 \leq j \leq \infty,
$$

is the $j$ th derivative of $y$ with respect to $x$.

(2) The notation

$$
a_{i j}:(1=1, n ; j=1, m)
$$

is used to denote the expression

$$
a_{i j} \text { for } i=1 \text { to } n \text { and } j=1 \text { to } m \text {. }
$$

(3) Let $\alpha_{k, i}=0:(k=1, n ; i=1, n)$ be a system of non-zero linear differential equations of the form

$$
\alpha_{k, i}=\sum_{j=0}^{N_{k, i}-1} a_{k, i, j+1} y^{(j)}=0:(k=1, n ; i=1, n)
$$

having coefficients $a_{k, i, j+1}:\left(k=1, n ; i=1, j=0, N_{k, i}-1\right)$ belonging to some differential field $F$ with field of constants $C$. A differential field containing $F$ is called a Picard-Vessiot extension of $F$ for $\alpha_{k, i}:(k=1, n ; i=$ $1, n)$ if

(a) $M=F\left\langle y_{1,1,1}, y_{1,1,2}, \ldots, y_{n, n, N_{n, n}}\right\rangle$ where $y_{k, i, 1}, y_{k, i, 2}, \ldots$, $y_{k, i}, N_{n, n}$ are $N_{k, i}$ solutions of (3) for $\left(k^{n}=1, n ; i=1, n\right)$ which are linearly independent over $C$, and

(b) $M$ has the same field of constants as $F$, that is, $M$ has the field of constants $C$.

(4) If $F$ is a differential field of charactristic zero then a non-zero differential polynomial $R$ over $F$ is an expression of the form

$$
R\left(y_{i}^{(j)}:(i=1, n ; j=0, m)\right)
$$

where $y_{1}(x), y_{2}(x), \ldots, y_{n}(x)$ are differential indeterminates, $n$ and $m$ are non-negative integers and

$$
R\left(y_{i}^{(j)}:(i=1, n ; j=0, m)\right)
$$

is a polynomial in the $n(m+1)$ variables

$$
y_{i}^{(j)}:(i=1, n ; j=0, m)
$$

having coefficients in $F$. 
(5) Let $f=f(x)$ be a function of $x$. Then $\operatorname{Ord}_{a} f$ is the order of vanishing of $f$ about a finite point $x=a$. If $f$ is written as a power series about $x=a$ in the following way,

$$
f=\sum_{-\infty}^{\infty} c_{i}(x-a)^{i}
$$

then $\operatorname{Ord}_{a} f$ is the smallest $i$ for which $c_{i} \neq 0$ if such an $i$ exists. Otherwise we say that $\operatorname{Ord}_{a} f=-\infty$.

\section{The theorem}

THEOREM 1. Let $K$ denote an algebraically closed field of characteristic zero. Suppose

$$
L_{1}(\mathbf{P}), L_{2}(\mathbf{P}), \ldots, L_{n}(\mathbf{P})
$$

are linearly independent linear forms in

$$
\mathbf{P}=\left(P_{1}(x), P_{2}(x), \ldots, P_{n}(x)\right)
$$

with coefficients

$$
f_{k, i}=f_{k, i}(x):(k=1, n ; i=1, n)
$$

where

(1) $P_{i}(x)$ is a polynomial in $x$ with coefficients in $K:(i=1, n)$ and

(2) $f_{k, i}(x)$ is a formal power series solution about $x=0$ of a non-zero linear differential equation

$$
\alpha_{k, i}=\alpha_{k, i}\left(\left(f_{k, i}\right),\left(f_{k, i}\right)^{(1)},\left(f_{k, i}\right)^{(2)}, \ldots,\left(f_{k, i}\right)^{\left(N_{k, i}\right)}\right)=0
$$

with coefficients in $K(x):(k=1, n ; i=1, n)$. Let Ord $_{a}$ denote the order of vanishing about a regular (finite) point of functions $f_{k, i}:(k=1, n ; i=$ $1, n)$. Then for each $\varepsilon>0$ either

$$
\operatorname{Ord}_{a}\left(L_{1} L_{2} \cdots L_{n}\right) \leq(1+\varepsilon) \sum_{i=1}^{n} \operatorname{deg} P_{i}+C
$$

where $C$ is an effectively computable constant (that is, it is independent of $\mathbf{P})$, or

$$
\mathbf{P}=\left(P_{1}(x), P_{2}(x), \ldots, P_{n}(x)\right)
$$

lies inside one of a finite number of proper subspaces of $(K(x))^{n}$.

The proof of Theorem 1 relies upon the construction of a differential polynomial

$$
D_{N}\left(P_{1}, P_{2}, \ldots, P_{n}\right)
$$


and, by proving a series of lemmas, finding upper and lower bounds on the quantity

$$
\operatorname{Ord}_{a} D_{N}\left(P_{1}, P_{2}, \ldots, P_{n}\right) \text {. }
$$

Let $M$ be the Picard-Vessiot extension of $K(x)$ corresponding to the differential equations $\alpha_{k, i}=0:(k=1, n ; i=1, n)$. that is $M$ is the minimal differential extension of $K(x)$ containing $f_{k, i, r}:(k=1, n ; i=$ $\left.1, n ; r=1, N_{k, i}\right)$ with the field of constants $K$. For $N \geq 0$ we denote by $M_{N}$ the vector space over $K$ generated by monomials of the form

$$
\prod_{k=1}^{n} \prod_{i=1}^{n} \prod_{r=1}^{N_{k, i}} f_{k, r, r}^{m_{k, i, r}} \quad \text { where } \sum_{k=1}^{n} \sum_{i=1}^{n} \sum_{r=1}^{N_{k, i}} m_{k, i, r}=N
$$

According to Hilbert's Theorem, later generalised by Serre and henceforth referred to as the Serre-Hilbert Theorem [3], $\operatorname{dim}_{k} M_{N}=P_{0}(N)$ for $N \geq N_{0}$, where $P_{0}(N)$ is an integer valued polynomial. We define $\mu_{N}$ to be equal to $\operatorname{dim}_{k} M_{N}$ and we take functions

$$
f_{j}^{N}:\left(j=1, \mu_{N}\right)
$$

as a basis of $M_{N}$, and introduce the following auxillary polynomial in the differential indeterminates $P_{1}, P_{2}, \ldots, P_{n}$ :

$$
D_{N}\left(P_{1}, P_{2}, \ldots, P_{n}\right)=\frac{W\left(P_{i} f_{f}^{N}:\left(i=1, n ; j=1, \mu_{N}\right)\right)}{\left(W\left(f_{j}^{N}:\left(j=1, \mu_{N}\right)\right)\right.}
$$

where

$$
W\left(h_{1}, h_{2}, \ldots, h_{m}\right)=\operatorname{det}\left(\left(\left(\frac{\partial}{\partial x}\right)^{l-1} h_{s}\right):(l=1, m ; s=1, m)\right) .
$$

Theorem 2. (1) $D_{N}\left(P_{1}, P_{2}, \ldots, P_{n}\right)$ is a differential polynomial in $P_{1}$, $P_{2}, \ldots, P_{n}$,

(2) the coefficients of the differential polynomial are invariant under the action of the differential Galois group of $M$ over $K(x)$ and so the coefficients belong to $K(x)$, and

(3) $\operatorname{Ord}_{a} D_{N}\left(P_{1}, P_{2}, \ldots, P_{n}\right) \leq \mu_{N} \sum_{i=1}^{n} \operatorname{deg} P_{i}$.

Since the wronskian is formed by taking the sum of products consisting of elements taken from each row, (1) is clearly true. We prove (2) by using the following two lemmas.

LEMMA 1. The coefficients of $D_{N}\left(P_{1}, P_{2}, \ldots, P_{n}\right)$ consist of the sum of terms of the form

$$
\frac{D_{1} \times D_{2} \times \cdots \times D_{n}}{(\bar{W})_{n}}
$$


where $D_{i}$ is a determinant of the form

for $i=1, n$ and where

$$
\left|\begin{array}{cccc}
\left(f_{1}^{N}\right)^{\left(v_{i, 1}-1\right)} & \left(f_{2}^{N}\right)^{\left(v_{i, 1}-1\right)} & \ldots & \left(f_{\mu_{N}}^{N}\right)^{\left(v_{i, 1}-1\right)} \\
\left(f_{1}^{N}\right)^{\left(v_{i, 2}-1\right)} & \left(f_{2}^{N}\right)^{\left(v_{i, 2}-1\right)} & \ldots & \left(f_{\mu_{N}^{N}}^{N}\right)^{\left(v_{i, 2}-1\right)} \\
\left(f_{1}^{N}\right)^{\left(v_{i, 3}-1\right)} & \left(f_{2}^{N}\right)^{\left(v_{i, 3}-1\right)} & \ldots & \left(f_{\mu_{N}}^{N}\right)^{\left(v_{i, 3}-1\right)} \\
\vdots & \vdots & & \vdots \\
\left(f_{1}^{N}\right)^{\left(v_{i, \mu_{N}}-1\right)} & \left(f_{2}^{N}\right)^{\left(v_{i, \mu_{N}}-1\right)} & \ldots & \left(f_{\mu_{N}}^{N}\right)^{\left(v_{i, \mu_{N}}-1\right)}
\end{array}\right|
$$

$$
\bar{W}=W\left(f_{1}^{N}:\left(j=1, \mu_{N}\right)\right) .
$$

Lemma 1 follows from the application and manipulation of the definition of deteminant.

LEMMA 2. Under the action of the differential Galois group of $M$ over $K(x)$, any determinant of the form

$$
D=\left|\begin{array}{cccc}
\left(f_{1}^{N}\right)^{\left(k_{1}\right)} & \left(f_{2}^{N}\right)^{\left(k_{1}\right)} & \ldots & \left(f_{\mu_{N}}^{N}\right)^{\left(k_{1}\right)} \\
\left(f_{1}^{N}\right)^{\left(k_{2}\right)} & \left(f_{2}^{N}\right)^{\left(k_{2}\right)} & \ldots & \left(f_{\nu_{N}}^{N}\right)^{\left(k_{2}\right)} \\
\left(f_{1}^{N}\right)^{\left(k_{3}\right)} & \left(f_{2}^{N}\right)^{\left(k_{3}\right)} & \ldots & \left(f_{\mu_{N}}^{N}\right)^{\left(k_{3}\right)} \\
\vdots & \vdots & & \vdots \\
\left(f_{1}^{N}\right)^{\left(k_{\mu_{N}}\right)} & \left(f_{2}^{N}\right)^{\left(k_{\mu_{N}}\right)} & \ldots & \left(f_{\mu_{N}}^{N}\right)^{\left(k_{\mu_{N}}\right)}
\end{array}\right|
$$

is sent to some constant times itself.

Under the action of the differential Galois group, $G$, of $M$ over $K(x)$, each $f_{j}^{N}$ is sent to a linear combination of $f_{1}^{N}, f_{2}^{N}, \ldots, f_{\mu_{N}}^{N}$ in the following way: for any automorphism $\Gamma \in G$,

$$
\Gamma: f_{r}^{N} \rightarrow \sum_{j=1}^{\mu_{N}} c_{r, j} f_{j}^{N}
$$

and

$$
\Gamma:\left(f_{r}^{N}\right)^{\left(k_{t}\right)} \rightarrow \sum_{j=1}^{\mu_{N}} c_{r, j}\left(f_{j}^{N}\right)^{\left(k_{t}\right)} \quad \text { for } t=1, \mu_{N},
$$

where $c_{r, j} \in K:\left(r=1, \mu_{N} ; j+1, \mu_{N}\right)$. Lemma 2 follows from the application of the above results to each element of $D$ and from the algebraic transformation of determinants.

By Lemma 1 , the coefficients of $D_{N}\left(P_{1}, P_{2}, \ldots, P_{n}\right)$ consist of the sum of terms of the form

$$
\frac{D_{1} \times D_{2} \times \cdots \times D_{n}}{(\bar{W})^{n}}
$$


which, applying Lemma 2 , are clearly invariant under the action of the differential Galois group $G$ and hence belong to $K(x)$. Hence $D_{N}\left(P_{1}, P_{2}, \ldots, P_{n}\right)$ is a polynomial in $P_{1}, P_{1}^{(1)}, \ldots, P_{n}^{\left(\mu_{N}-1\right)}$ having terms of the form

$$
A_{\bar{a}} \prod_{i=1}^{n} \prod_{j=1}^{\mu_{N}} P_{i}^{\left(a_{i, j}\right)}
$$

where

$$
A_{\bar{a}} \in K(x) \text { and } \sum_{i=1}^{n} \sum_{j=1}^{\mu_{N}} a_{i, j} \leq n \mu_{N-1} .
$$

Therefore

$$
\operatorname{Ord}_{a} D_{N}\left(P_{1}, P_{2}, \ldots, P_{n}\right) \leq \mu_{N} \sum_{i=1}^{n} \operatorname{deg} P_{i}+C_{1}(N)
$$

where $C_{1}(N)$ is a constant independent of $P_{1}, P_{2}, \ldots, P_{n}$.

LEMMA 3. If $f_{l}^{N-1}$ belongs to the basis of $M_{N-1}$ and $f_{k, i}$ is a solution of one of the original differential equations $\alpha_{k, i}$, then

$$
f_{l}^{N-1} \sum_{i=1}^{n} P_{i} f_{k, i}=\sum_{i=1}^{n} P_{i} \sum_{j=1}^{\mu_{N}} C_{l}^{i, j} f_{j}^{N}
$$

where the $C_{l}^{i, j}$ are constants belonging to $K$ and the vectors $C_{l}=C_{l}^{i, j}:(i=$ $\left.1, n ; j=1, \mu_{n}\right)$ are linearly independent over $K$.

Clearly $M_{1} M_{n-1} \subset M_{N}$ and so

$$
f_{k, i} f_{l}^{N-1} \in M_{N}
$$

It follows that

$$
f_{k, i} f_{l}^{N-1}=\sum_{j=1}^{\mu_{N}} C_{l}^{i, j} f_{j}^{N}
$$

where $C_{l}^{i, j} \in K:\left(i=1, n ; j=1, \mu_{N}\right)$ for $l=1, \mu_{N-1}$. Multiplying by $P_{i}$ and summing for $i=1, \ldots, n$ gives the required result. The vectors $C_{l}=C_{l}^{i, j}:\left(i=1, n ; j=1, \mu_{N}\right)$ are clearly linearly independent over $K$, for suppose on the contrary that there are constants $d_{l} \in K$ not all zero such that

$$
\sum_{l=1}^{\mu_{N-1}} C_{l} d_{l}=0
$$


Then

$$
\sum_{l=1}^{\mu_{N-1}} C_{l}^{i, j} d_{l}=0 \text { for }\left(i=1, n ; j=1, \mu_{N}\right) .
$$

Multiplying by $f_{j}^{N}$ and summing for $j=1, \ldots, \mu_{N}$ we see that

$$
\sum_{l=1}^{\mu_{N-1}} d_{l} \sum_{j=1}^{\mu_{N}} C_{l}^{i, j} f_{j}^{N}=0
$$

Hence

$$
\sum_{l=1}^{\mu_{N-1}}\left(d_{l} f_{k, i}\right) f_{l}^{N-1}=f_{k, i} \sum_{l=1}^{\mu_{N-1}} d_{l} f_{l}^{N-1}=0
$$

implying that

$$
\sum_{l=1}^{\mu_{N-1}} d_{l} f^{N-1} / l=0
$$

which is a contradiction of the linear independence of the $f_{l}^{N-1}$ over $K$. Hence the $C_{l}^{i, j}:\left(l=1, \mu_{N-1}\right)$ are linearly independent over $K$.

LEMMA 4. We can make a non-singular transformation that reduces the determinant

$$
W=W\left(P_{i} f_{j}^{N}:\left(i=1, n ; j=1, \mu_{N}\right)\right)
$$

to the form $\hat{W}$, the determinant of the matrix $\hat{A}$ in which the first $\mu_{N-1}$ columns have the form

$$
\left(\left(\frac{\delta}{\delta x}\right)^{(s-1)}\left\{\sum_{i=1}^{n} \sum_{j=1}^{\mu_{N}} P_{i} f_{j}^{N} C_{l}^{i, j}\right\} s=1, n \mu_{N}\right)
$$

for $l=1, \mu_{N-1}$ or

$$
\left(\left(\frac{\delta}{\delta x}\right)^{(s-1)}\left\{f_{l}^{(N-1)} \sum_{i=l}^{n} P_{i} f_{k, i}\right\} s=1, n \mu_{N}\right)
$$

for $l=1, \mu_{N-1}$.

Lemma 4 follows if we can make a non-singular transformation that reduces $W$ to the form $W_{k}$, the determinant of the matrix $A_{k}$ in which the first $k$ columns have the above form, for $k=1, \mu_{N-1}$. This is proved using induction on $k$. 
LEMMA 5. The equations

$$
\prod_{\lambda=0}^{\left(n \mu_{N}-1\right)}\left(\frac{\partial}{\partial P_{n}^{(\lambda)}}\right)^{e_{k, \lambda+1}} D_{N}\left(P_{1}, P_{2}, \ldots, P_{n}\right)=0
$$

are satisfied whenever

$$
\sum_{\lambda=0}^{\left(n \mu_{N}-1\right)} e_{k, \lambda+1}<\mu_{N-1}
$$

at

$$
\sum_{i=1}^{n} P_{i} f_{k, i}=0
$$

for $k=1, n$.

Clearly, under the transformation of Lemma 4, the above equations are all satisfied if and only if

$$
\prod_{\lambda=0}^{\left(n \mu_{N}-1\right)}\left(\frac{\partial}{\partial P_{n}^{(\lambda)}}\right)^{e_{k, \lambda+1}} \hat{W}=0
$$

whenever

$$
\sum_{\lambda=0}^{\left(n \mu_{N}-1\right)} e_{k, \lambda+1}<\mu_{N-1}
$$

at

$$
\sum_{i=l}^{n} P_{i} f_{k, i}=0
$$

for $k=1, n$, which follows since each of the derivatives of $\hat{W}$ consists of the sum of determinants, each having at least one of their first $\mu_{N-1}$ columns identical to that of $\hat{W}$. Hence at

$$
\sum_{i=1}^{n} P_{i} f_{k, i}=0
$$

the expression

$$
\prod_{\lambda=0}^{\left(n \mu_{N}-1\right)}\left(\frac{\partial}{\partial P_{n}^{(\lambda)}}\right)^{e_{k, \lambda+1}} \hat{W} \quad\left(\text { where } \sum_{\lambda=0}^{\left(n \mu_{N}-1\right)} e_{k, \lambda+1}<\mu_{N-1}\right)
$$

consists of the sum of determinants, each of which has at least one column consisting entirely of zeros. 
LEMMa 6. (1) $D_{N}\left(P_{1}, P_{2}, \ldots, P_{n}\right)$ can be expressed as the sum of monomials each of which has the form

$$
A_{\bar{e}} \prod_{k=1}^{n} \prod_{\lambda=0}^{\left(n \mu_{N}-1\right)}\left(\left\{\frac{L_{k}}{f_{k, n}}\right\}^{(\lambda)}\right)^{e_{k, \lambda+1}}
$$

where

$$
\sum_{\lambda=0}^{\left(n \mu_{N}-1\right)} e_{k, \lambda+1} \geq \mu_{N-1} \quad \text { for } k=1, n,
$$

where

$$
\bar{e}=\left\{e_{1,1}, e_{1,2}, \ldots, e_{n, n \mu_{N}}\right\}
$$

and where for each set $\bar{e}$,

$$
A_{\bar{e}}=A_{\bar{e}}\left(f_{k, i}^{(\lambda)}:\left(k=1, n ; i=1, n ; \lambda=0,\left(n \mu_{N}-1\right)\right)\right)
$$

is some polynomial in

$$
f_{k, i}^{(\lambda)}:\left(k=1, n ; i=1, R ; \lambda=0, n \mu_{N}-1\right) .
$$

(2) If $D_{N}\left(P_{1}, P_{2}, \ldots, P_{n}\right)$ is non-zero, then

$$
\left.\operatorname{Ord}_{a} D_{N}\left(P_{1}, P_{2}, \ldots, P_{n}\right) \geq \mu_{N-1} \operatorname{Ord}_{a} L_{1} L_{2} \cdots L_{n}\right)-C_{3}(N)
$$

where $C_{3}(N)$ is a constant depending only on $N$ and $f_{j}^{N}:\left(j=1, \mu_{N}\right)$.

Part (1) follows from the Taylor expansion of $D_{N}\left(P_{1}, P_{2}, \ldots, P_{n}\right)$ about $L_{1}=0, L_{2}=0, \ldots, L_{n}=0$ and Lemma 5. Part (2) follows from (1) and the properties of $\operatorname{Ord}_{a}$.

Proof of Theorem 1. From (4) and (5) we see, if $D_{N}$ is non-zero, that

$$
\mu_{N} \sum_{i=1}^{n} \operatorname{deg} P_{i}+C_{1}(N) \geq \mu_{N-1} \operatorname{Ord}_{a}\left(L_{1} L_{2} \cdots L_{n}\right)-C_{3}(N)
$$

and so

$$
\operatorname{Ord}_{a}\left(L_{1} L_{2} \cdots L_{n}\right) \leq \frac{\mu_{N}}{\mu_{N-1}} \sum_{i=1}^{n} \operatorname{deg} P_{i}+C_{4}(N) .
$$

Now, according to Hilbert's Theorem [3],

$$
\frac{\mu_{N}}{\mu_{N-1}}=\frac{\operatorname{dim}_{k} M_{N}}{\operatorname{dim}_{k} M_{N-1}} \rightarrow 1 \text { as } N \rightarrow \infty \text {. }
$$

Hence, if $D_{N}\left(P_{1}, P_{2}, \ldots, P_{n}\right) \neq 0$, for $N \geq N_{1}(\varepsilon)$, we have

$$
\operatorname{Ord}_{a}\left(L_{1} L_{2} \cdots L_{n}\right) \leq(1+\varepsilon) \sum_{i=1}^{n} \operatorname{deg} P_{i}+C_{4}(N),
$$


where $C_{4}(N)$ is a constant independent of $P_{1}, P_{2}, \ldots, P_{n}$. However, if $D_{N}\left(P_{1}, P_{2}, \ldots, P_{n}\right)=0$ the functions $P_{i} f_{j}^{N}:\left(i=1, n ; j=1, \mu_{N}\right)$ are linearly dependent over $K$. Hence

$$
\sum_{i=1}^{n} P_{i}\left(\sum_{j=1}^{\mu_{N}} c_{i, j} f_{j}^{N}\right)=0 \text { for constants } c_{i, j} \in K \text { not all zero. }
$$

Choosing a basis of $M_{N}$ over $K(x), g_{r}^{N}:\left(r=1, \alpha_{N}\right)$ say, having dimension $\alpha_{N}$, each of the $f_{j}^{N}$ can be expressed as a linear combination of the $g_{r}^{N}:\left(r=1, \alpha_{N}\right)$ having coefficients in $K(x)$ with degrees bounded by some $m=m(N)$. That is,

$$
f_{j}^{N}=\sum_{r=1}^{\alpha_{N}} a_{r, j}(x) g_{r}^{N}:\left(j=1, \mu_{N}\right)
$$

and so

$$
\begin{aligned}
\sum_{j=1}^{\mu_{N}} c_{i, j} f_{j}^{N} & =\sum_{j=1}^{\mu_{N}} \sum_{r=1}^{\alpha_{N}} c_{i, j} a_{r, j}(x) g_{r}^{N} \\
& =\sum_{r=1}^{\alpha_{N}}\left(\sum_{j=1}^{\mu_{N}} c_{i, j} a_{r, j}(x)\right) g_{r}^{N} \\
& =\sum_{r=1}^{\alpha_{N}} b_{r, i}(x) g_{r}^{N}
\end{aligned}
$$

where the $b_{r, i}(x) \in K(x)$ with degrees bounded by $m$, not all zero. Now as we saw above,

$$
\sum_{i=1}^{n} P_{i}\left(\sum_{j=1}^{\mu_{N}} c_{i, j} f_{j}^{N}\right)=0
$$

Hence

$$
\sum_{i=1}^{n} P_{i}\left(\sum_{r=1}^{\alpha_{N}} b_{r, i}(x) g_{r}^{N}\right)=0
$$

and so

$$
\sum_{r=1}^{\alpha_{N}} g_{r}^{N}\left(\sum_{i=1}^{n} P_{i} b_{r, i}(x)\right)=0
$$

At least one of the linear relations

$$
\sum_{i=1}^{n} P_{i} b_{r, i}(x)
$$


is non-trivially zero, by the linear independence of the $g_{r}^{N}$ over $K(x)$. That is, there exists at least one linear relation among the $P_{i}$ of the form

$$
B_{1}(x) P_{1}(x)+B_{2}(x) P_{2}(x)+\cdots+B_{n}(x) P_{n}(x)=0,
$$

where $B_{i}(x) \in K(x):(i=1, n)$ and where

$$
\operatorname{deg} B_{i}(x) \leq m^{\prime}=m^{\prime}(N):(i=1, n) .
$$

Because the degrees of the polynomials $B_{i}(x):(i=1, n)$ are bounded, only finitely many linear relations of the above form exist. Now the expressions

$$
B_{1}(x) P_{1}(x)+B_{2}(x) P_{2}(x)+\cdots+B_{n}(x) P_{n}(x)=0
$$

imply that at most $(n-1)$ of $P_{1}, P_{2}, \ldots, P_{n}$ are linearly independent, and hence from (6) and the above, either

$$
\operatorname{Ord}_{a}\left(L_{1} L_{2} \cdots L_{n}\right) \leq(1+\varepsilon) \sum_{i=1}^{n} \operatorname{deg} P_{i}+C_{4}(N)
$$

or the polynomials $P_{1}, P_{2}, \ldots, P_{n}$ lie inside a finite number of rational subspaces of $(K(x))^{n}$.

In considering a possible extension of Theorem 1 to obtain an upper bound for

$$
\prod_{d \in A} \operatorname{Ord}_{d}\left(L_{1} L_{2} \cdots L_{n}\right)
$$

where $A=\left\{a_{1}, a_{2}, \ldots, a_{r}, \infty\right\}$ and where $a_{1}, a_{2}, \ldots, a_{r}$ are elements of $K$, it seems difficult to approach this type of result using the homogeneous form of the wronskian. It is the inclusion of the point $x=\infty$ in $A$ that is responsible for this lack of conformity. One might also expect that one could put $\varepsilon=0$ in Theorem 1 .

\section{References}

[1] D. V. Chudnovsky and G. V. Chudnovsky, 'Rational approximations to solutions of linear differential equations', Proc. Nat. Acad. Sci. U.S.A. 80 (1983), 5158-5162.

[2] J. H. Evertse and K. Györy, ' $S$-unit equations and their applications', New advances in transcendence theory (Durham, 1986), pp. 110-174, (Cambridge University Press, Cambridge, New York, 1988).

[3] N. Jacobson, Basic algebra II, (Freeman, 1980).

[4] C. F. Osgood, 'Concerning a possible Thue-Siegel-Roth theorem for algebraic differential equations', Number theory and algebra, pp. 223-234, (Academic Press, 1977).

[5] C. F. Osgood, 'Sometimes effective Thue-Siegel-Roth-Schmidt-Nevanlinna bounds, or better', J. Number Theory 21 (1985), 347-389.

[6] K. F. Roth, 'Rational approximations to algebraic numbers', Mathematika 2 (1955), $1-20$. 
[7] H. P. Schlickerwei, 'On products of special linear forms with algebraic coefficients', Acta Arith. 31 (1976), 389-398.

[8] W. M. Schmidt, 'Diophantine approximations', Lecture Notes in Mathematics 785, (Springer-Verlag, 1980).

University of Western Australia

Nedlands WA 6009

Australia 